\title{
PROPERTIES OF THE MODIFIED CAPUTO'S DERIVATIVE OPERATOR FOR CERTAIN ANALYTIC FUNCTIONS
}

\author{
Jamal Y. Salah \\ Department of Basic Sciences \\ College of Applied Sciences \\ A'Sharqiyah University \\ Ibra, OMAN
}

\begin{abstract}
In this paper, a class $A_{\eta, \lambda}(\alpha, \beta, \gamma)$ of analytic functions involving the integral operator $J_{\eta, \lambda} f(z)=z+\sum_{n=2}^{\infty} \frac{(\Gamma(n+1))^{2} \Gamma(2+\eta-\lambda) \Gamma(2-\eta)}{\Gamma(n+\eta-\lambda+1) \Gamma(n-\eta+1)} a_{n} z^{n}$, given by Salah and Darus in [5] is defined. The extreme points for this class are provided, the coefficient bounds and radii of univalency and starlikeness are also provided.
\end{abstract}

AMS Subject Classification: 30C45, 30C50

Key Words: Caputo's differentiation operator, univalency, starlikeness, Hadamard product

\section{Introduction and Preliminaries}

Let $A$ denote the class of functions of the form $f(z)=z+\sum_{n=2}^{\infty} a_{n} z^{n}$ which are analytic in the unit disk $U=\{z:|z|<1\}$. Also let $S$ denote the familiar subclass of $A$ consisting of all functions which are univalent in $U$.

$\begin{array}{lr}\text { Received: } & \text { June 24, } 2016 \\ \text { Revised: } & \text { August } 31,2016 \\ \text { Published: } & \text { September 30, } 2016\end{array}$

(c) 2016 Academic Publications, Ltd. url: www.acadpubl.eu 
Definition 1.1. The fractional integral of order $\lambda$ is defined, for a function $f(z) \in A$, by

$$
D_{z}^{-\lambda} f(z)=\frac{1}{\Gamma(\lambda)} \int_{0}^{z} \frac{f(t)}{(z-t)^{1-\lambda}} d t \quad(\lambda>0),
$$

where the multiplicity of $(z-t)^{\lambda-1}$ is removed by requiring $\log (z-t)$ to be real when $z-t>0$,

Definition 1.2. The fractional derivative of order $\lambda$ is defined, for a function $f(z) \in A$, by

$$
D_{z}^{\lambda} f(z)=\frac{1}{\Gamma(1-\lambda)} \frac{d}{d z} \int_{0}^{z} \frac{f(t)}{(z-t)^{\lambda}} d t \quad(0 \leq \lambda<1),
$$

where the multiplicity of $(z-t)^{-\lambda}$ is removed by requiring $\log (z-t)$ to be real when $z-t>0$.

Definition 1.3. The Caputo's definition of fractional-order derivative is given by

$$
D^{\alpha} f(t)=\frac{1}{\Gamma(n-\alpha)} \int_{a}^{t} \frac{f^{(n)}(\tau)}{(t-\tau)^{\alpha+1-n}} d \tau
$$

where $n-1<\operatorname{Re}(\alpha) \leq n, n \in N$ and $\alpha$ is allowed to be real or complex number, $\mathrm{a}$ is the initial value of the function $\mathrm{f}$.

Definition 1.4. The Modified Caputo's derivative operator is given by

$$
J_{\eta, \lambda} f(z)=\frac{\Gamma(2+\eta-\lambda)}{\Gamma(\eta-\lambda)} z^{\lambda-\eta} \int_{0}^{z} \frac{\Omega^{\eta} f(t)}{(z-t)^{\lambda+1-\eta}} d t
$$

where $\eta$ (real number), $(\eta-<\lambda \leq \eta<2)$, and $\Omega^{\eta} f(t)=\Gamma(2-\eta) t^{\eta} D_{t}^{\eta} f(t)$.

Now, if $z+\sum_{n=2}^{\infty} a_{n} z^{n}$ is an analytic function in $\mathrm{A}$, then

$$
J_{\eta, \lambda} f(z)=z+\sum_{n=2}^{\infty} \frac{(\Gamma(n+1))^{2} \Gamma(2+\eta-\lambda) \Gamma(2-\eta)}{\Gamma(n+\eta-\lambda+1) \Gamma(n-\eta+1)} a_{n} z^{n} .
$$

In this paper, we consider the following subclass of $A$

$$
A_{\eta, \lambda}(\alpha, \beta, \gamma)
$$




$$
=\left\{f(z) \in A: R\left\{\alpha \frac{J_{\eta, \lambda} f(z)}{z}+\beta\left(J_{\eta, \lambda} f(z)\right)^{\prime}\right\}>\gamma(z \in U)\right\},
$$

for some real $\alpha, \beta>0$, and $\gamma$ with $0 \leq \gamma \leq \alpha+\beta \leq 1$. Where for $\eta$ (real number) and $(\eta-1<\lambda \leq \eta<2)$.

Note that $J_{0,0} f(z)=f(z)$. Thus the class $A_{\eta, \lambda}(\alpha, \beta, \gamma)$ is a generalization of the class:

$$
A(\alpha, \beta, \gamma)=\left\{f(z) \in A: R\left\{\alpha \frac{f(z)}{z}+\beta(f(z))^{\prime}\right\}>\gamma(z \in U)\right\} .
$$

The class $(1,7)$ was introduced and studied by Zhi-Gang Wang,Chun-Yi Gao and Shao-Mou Yuan [1]. While Saitoh [2] and Owa [3, 4] have determined and discussed certain related properties in a special case where $\alpha=1-\beta$.

In the present work, first we determine the extreme points of (1.6), then we find the coefficient bounds and radius of univalency for functions belonging to this class.

\section{Extreme Points of the Class $A_{\eta, \lambda}(\alpha, \beta, \gamma)$}

Theorem 2.1. A function $f(z) \in A_{\eta, \lambda}(\alpha, \beta, \gamma)$ if and only if $f(z)$ can be expressed as

$$
\begin{aligned}
f(z)=z+ & \frac{2(\alpha+\beta-\gamma)}{\Gamma(2-\eta) \Gamma(2+\eta-\lambda)} \\
& \int_{|x|=1}\left(\sum_{n=2}^{\infty} \frac{\Gamma(n+\eta-\lambda+1) \Gamma(n-\eta+1) x^{n-1}}{(\Gamma(n+1))^{2}(\alpha+n \beta)} z^{n}\right) d \mu(x),
\end{aligned}
$$

where $\mu(x)$ is the probability measure on $X=\{x:|x|=1\}$. For fixed $\alpha, \beta$ and $\gamma, A(\alpha, \beta, \gamma)$ and the probability measures $\{\mu\}$ defined on $X$ are one-to-one by the expression (2.1).

Proof. By the definition of $A_{\eta, \lambda}(\alpha, \beta, \gamma)$, we know $f(z) \in A_{\eta, \lambda}(\alpha, \beta, \gamma)$ if and only if

$$
\frac{\alpha\left(\frac{J_{\eta, \lambda} f(z)}{z}\right)+\beta\left(J_{\eta, \lambda} f(z)\right)^{\prime}-\gamma}{\alpha+\beta-\gamma} \in P,
$$

where $P$ denotes the normalized class of analytic functions which have positive real part. Using Herglotz expressions of functions in $P$, we have

$$
\frac{\alpha\left(\frac{J_{\eta, \lambda} f(z)}{z}\right)+\beta\left(J_{\eta, \lambda} f(z)\right)^{\prime}-\gamma}{\alpha+\beta-\gamma}=\int_{|x|=1} \frac{1+x z}{1-x z} d \mu(x)
$$


or

$$
\frac{\alpha J_{\eta, \lambda} f(z)}{\beta z}+\left(J_{\eta, \lambda} f(z)\right)^{\prime}=\frac{1}{\beta} \int_{|x|=1} \frac{\alpha+\beta+(\alpha+\beta-2 \gamma) x z}{1-x z} d \mu(x) .
$$

Thus we have

$$
\begin{aligned}
& z^{-\frac{\alpha}{\beta}} \int_{0}^{z}\left[\frac{\alpha J_{\eta, \lambda} f(\zeta)}{\beta \zeta}+\left(J_{\eta, \lambda} f(\zeta)\right)^{\prime}\right] \zeta^{\frac{\alpha}{\beta}} d \zeta \\
& =\frac{1}{\beta} \int_{|x|=1}\left[z^{\frac{-\alpha}{\beta}} \int_{0}^{z} \frac{\alpha+\beta+(\alpha+\beta-2 \gamma) x \zeta}{1-x \zeta} \zeta^{\frac{\alpha}{\beta}} d \zeta\right] d \mu(x) .
\end{aligned}
$$

That is

$$
\begin{array}{r}
J_{\eta, \lambda} f(z) \\
=\frac{1}{\alpha+\beta} \int_{|x|=1}\left[(2 \gamma-\alpha-\beta) z+2(\alpha+\beta-\gamma) \sum_{n=0}^{\infty} \frac{(\alpha+\beta) x^{n} z^{n+1}}{(n+1) \beta+\alpha}\right] d \mu(x) \\
=z+\int_{|x|=1}\left(\sum_{n=2}^{\infty} \frac{2(\alpha+\beta-\gamma)}{\alpha+n \beta} x^{n-1} z^{n}\right) d \mu(x) .
\end{array}
$$

The last expression is equivalent to

$$
\begin{aligned}
f(z)=z+ & \frac{2(\alpha+\beta-\gamma)}{\Gamma(2-\eta) \Gamma(2+\eta-\lambda)} \\
& \int_{|x|=1}\left(\sum_{n=2}^{\infty} \frac{\Gamma(n+\eta-\lambda+1) \Gamma(n-\eta+1) x^{n-1}}{(\Gamma(n+1))^{2}(\alpha+n \beta)} z^{n}\right) d \mu(x) .
\end{aligned}
$$

This deductive process can be conversed, so we have proved the first part of the theorem.

We know that both probability measures $\{\mu\}$ and class $P$, class $P$ and $A_{\eta, \lambda}(\alpha, \beta, \gamma)$ are one-to-one, so the second part of the theorem is true. This completes the proof.

Corollary 2.2. The extreme points of the class $A_{\eta, \lambda}(\alpha, \beta, \gamma)$ are

$$
\begin{aligned}
f_{x}(z)=z+ & \frac{2(\alpha+\beta-\gamma)}{\Gamma(2-\eta) \Gamma(2+\eta-\lambda)} \\
& \sum_{n=2}^{\infty} \frac{\Gamma(n+\eta-\lambda+1) \Gamma(n-\eta+1) x^{n-1}}{(\Gamma(n+1))^{2}(\alpha+n \beta)} z^{n} \quad(|x|=1) .
\end{aligned}
$$


Proof. Using the notation $f_{x}(z),(2.1)$ can be written as

$$
f_{\mu}(z)=\int_{|x|=1} f_{x}(z) d \mu(x)
$$

By Theorem (2.1) , the map $\mu \rightarrow f_{\mu}$ is one-to-one, so the assertion follows.

Corollary 2.3. If $f(z)=z+\sum_{n=2}^{\infty} a_{n} z^{n} \in A_{\eta, \lambda}(\alpha, \beta, \gamma)$, then for $n \geq 2$, we have

$$
\left|a_{n}\right| \leq \frac{2(\alpha+\beta-\gamma) \Gamma(n+\eta-\lambda+1) \Gamma(n-\eta+1)}{\Gamma(2-\eta) \Gamma(2+\eta-\lambda)(\Gamma(n+1))^{2}(\alpha+n \beta)} .
$$

Proof. The coefficient bounds are maximized at an extreme point so the result follows from $(2.2)$.

Corollary 2.4. If $f(z)=z+\sum_{n=2}^{\infty} a_{n} z^{n} \in A_{\eta, \lambda}(\alpha, \beta, \gamma)$, then for $|z|=$ $r<1$, we have

$$
|f(z)| \leq r+\frac{2(\alpha+\beta-\gamma)}{\Gamma(2-\eta) \Gamma(2+\eta-\lambda)} \sum_{n=2}^{\infty} \frac{\Gamma(n+\eta-\lambda+1) \Gamma(n-\eta+1) r^{n}}{(\Gamma(n+1))^{2}(\alpha+n \beta)}
$$

This result follows from (2.3).

\section{Radius of Univalency and Starlikeness}

Theorem 3.1. Let $f(z) \in A_{\eta, \lambda}(\alpha, \beta, \gamma)$, then $f(z)$ is univalent(close-toconvex) in $|z|<R(\alpha, \beta, \gamma)$, where

$$
R(\alpha, \beta, \gamma)=\inf _{n}\left\{\frac{(n \beta+\alpha)(\Gamma(n+1))^{2} \Gamma(2-\eta) \Gamma(2+\eta-\lambda)}{2 n(\alpha+\beta-\gamma) \Gamma(n+\eta-\lambda+1) \Gamma(n-\eta+1)}\right\}^{\frac{1}{n-1}}
$$

Proof. It suffices to show that

$$
\left|f^{\prime}(z)-1\right|<1
$$

For the left hand side of (3.1) we have

$$
\left|\sum_{n=2}^{\infty} n a_{n} z^{n-1}\right| \leq \sum_{n=2}^{\infty} n\left|a_{n}\right||z|^{n-1} .
$$


The last expression is less than 1 if

$$
|z|^{n-1}<\frac{(n \beta+\alpha)(\Gamma(n+1))^{2} \Gamma(2-\eta) \Gamma(2+\eta-\lambda)}{2 n(\alpha+\beta-\gamma) \Gamma(n+\eta-\lambda+1) \Gamma(n-\eta+1)} .
$$

To show that the bound $R(\alpha, \beta, \gamma)$ is the best possible,we consider the function $f(z) \in A$ defined by

$$
f(z)=z-\frac{\Gamma(n+\eta-\lambda+1) \Gamma(n-\eta+1) 2(\alpha+\beta-\gamma)}{(\Gamma(n+1))^{2}(\alpha+n \beta) \Gamma(2-\eta) \Gamma(2+\eta-\lambda)} z^{n} .
$$

If $\sigma>R(\alpha, \beta, \gamma)$, then there exists $n \geq 2$ such that

$$
\left\{\frac{(n \beta+\alpha)(\Gamma(n+1))^{2} \Gamma(2-\eta) \Gamma(2+\eta-\lambda)}{2 n(\alpha+\beta-\gamma) \Gamma(n+\eta-\lambda+1) \Gamma(n-\eta+1)}\right\}^{\frac{1}{n-1}}<\sigma .
$$

Since $f^{\prime}(0)=1>0$ and

$$
f^{\prime}(\sigma)=1-\frac{\Gamma(n+\eta-\lambda+1) \Gamma(n-\eta+1) 2 n(\alpha+\beta-\gamma)}{(\Gamma(n+1))^{2}(\alpha+n \beta) \Gamma(2-\eta) \Gamma(2+\eta-\lambda)} \sigma^{n-1}<0 .
$$

Thus, there exists $\sigma_{0} \in(0, \sigma)$ such that $f^{\prime}\left(\sigma_{0}\right)=0$, which implies that $f(z)$ is not univalent in $|z|<\sigma$. This completes the proof of Theorem 3.1.

Theorem 3.2. If $f(z) \in A_{\eta, \lambda}(\alpha, \beta, \gamma)$ then $f(z)$ is starlike of order $\mu$, $|z|<r_{0}, 0 \leq \mu<1$ where

$$
r_{0}=\inf _{n}\left\{\frac{(1-\mu) \Gamma(2-\eta) \Gamma(2+\eta-\lambda)(\Gamma(n+1))^{2}(n \beta+\alpha)}{2(n-\mu)(\alpha+\beta-\gamma) \Gamma(n+\eta-\lambda+1) \Gamma(n-\eta+1)}\right\}^{\frac{1}{n-1}}
$$

Proof. It suffices to show that

$$
\left|\frac{z f^{\prime}(z)}{f(z)}-1\right|<1-\mu .
$$

Using the same techniques of the previous theorem to proof of theorem (3.2) can be easily derived. 


\section{References}

[1] Zhi-Gang Wang, Chu-Yi Gao, Shao-Mou Yuan, On the univalency of certain analytic functions, J. Ineq.Pure and Appl. Math., 7, No. 1, Art. 9 (2006).

[2] H. Saitoh, On inequalities for certain analytic functions, Math. Japon, 35 (1990), 10731076.

[3] S. Owa, Some properties of certain analytic functions, Soochow J. Math., 13 (1987), 197-201.

[4] S. Owa, Generalization properties for certain analytic functions, Internat. J. Math. Math. Sci., 21 (1998), 707-712.

[5] J. Salah, M. Darus, A subclass of uniformly convex functions associated with fractional calculus operator involving Caputo's fractional differentiation, Acta. Univ. Apl., 24 (2010), 295-306. 
